\title{
Review Article \\ Structural Changes in Individual Retinal Layers in Diabetic Macular Edema
}

\author{
Tomoaki Murakami and Nagahisa Yoshimura \\ Department of Ophthalmology and Visual Sciences, Kyoto University Graduate School of Medicine, 54 Shogoin-Kawaracho, \\ Sakyo, Kyoto 606-8507, Japan \\ Correspondence should be addressed to Tomoaki Murakami; mutomo@kuhp.kyoto-u.ac.jp
}

Received 3 May 2013; Revised 8 July 2013; Accepted 10 July 2013

Academic Editor: Ahmed M. Abu El-Asrar

Copyright (C) 2013 T. Murakami and N. Yoshimura. This is an open access article distributed under the Creative Commons Attribution License, which permits unrestricted use, distribution, and reproduction in any medium, provided the original work is properly cited.

Optical coherence tomography (OCT) has enabled objective measurement of the total retinal thickness in diabetic macular edema (DME). The central retinal thickness is correlated modestly with visual impairment and changes paradoxically after treatments compared to the visual acuity. This suggests the clinical relevance of the central retinal thickness in DME and the presence of other factors that affect visual disturbance. Recent advances in spectral-domain (SD) OCT have provided better delineation of the structural changes and fine lesions in the individual retinal layers. Cystoid spaces in the inner nuclear layer and outer plexiform layer are related to quantitative and qualitative parameters in fluorescein angiography. OCT often shows vitreoretinal interface abnormalities in eyes with sponge-like retinal swelling. Serous retinal detachment is sometimes accompanied by hyperreflective foci in the subretinal fluid, which exacerbates the pathogenesis at the interface of the photoreceptors and retinal pigment epithelium. Photoreceptor damage at the fovea is thought to be represented by disruption of the external limiting membrane or the junction between the inner and outer segment lines and is correlated with visual impairment. Hyperreflective foci in the outer retinal layers on SD-OCT images, another marker of visual disturbance, are associated with foveal photoreceptor damage.

\section{Introduction}

Diabetic macular edema (DME), a leading cause of visual impairment in individuals of working age, is mediated by multiple and complex mechanisms in its pathogenesis [1-4]. Pathophysiology, that is, vascular hyperpermeability and ischemia, is represented by clinical findings seen on fluorescein angiography (FA) images [5-8]. Basic research has elucidated the molecular mechanisms including vascular endothelial growth factor in DME and proliferative diabetic retinopathy (PDR) [9-18]. Using biomicroscopy, clinical ophthalmologists observe thickening of the sensory retina and vascular lesions in DME. However, it is difficult to objectively evaluate the neuroglial changes in the retina. In contrast, histologic studies have reported that cystoid spaces are present mainly in the inner nuclear layer (INL) and the outer plexiform layer (OPL) and contribute partly to macular thickening [19-21]. Electron microscopy showed that, in addition to the accumulation of the extracellular fluids, intracytoplasmic swelling of the Müller cells might be a pathophysiologic mechanism in DME.

Optical coherence tomography (OCT) provides retinal sectional images as in histology study (Figure 1) and is useful for qualitative and quantitative evaluation of pathological retinal changes [22]. The original instrument, time-domain OCT, has been replaced with spectral-domain OCT (SDOCT), which has higher resolution and reduced speckle noise. Clinicians can appreciate the improved delineation of the fine pathological lesions and the clearer borders between the individual retinal layers.

This review summarizes the current understanding of the retinal thickness, pathomorphologies, and photoreceptor damage in DME that can be seen on OCT.

\section{Retinal Thickening on OCT Images}

Biomicroscopy allows ophthalmologists to subjectively evaluate retinal thickening, whereas OCT has enabled objective 


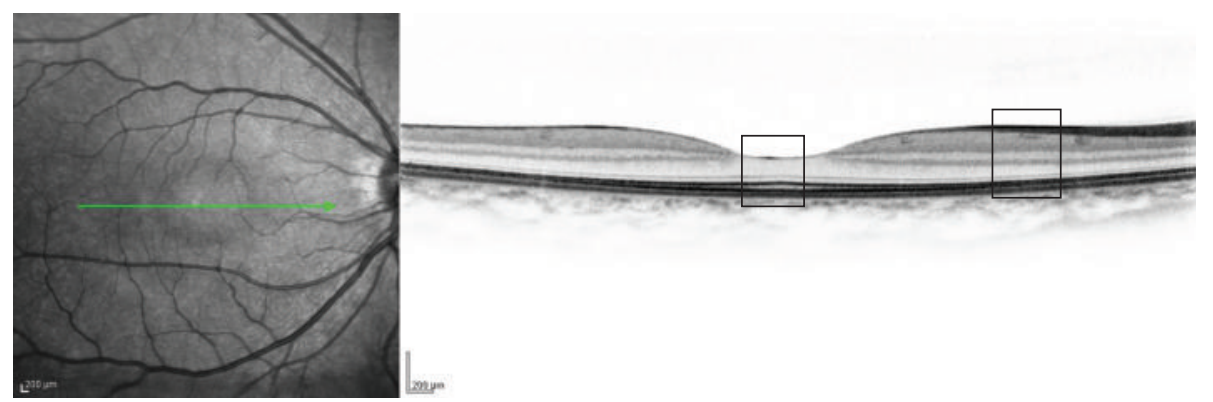

(a)

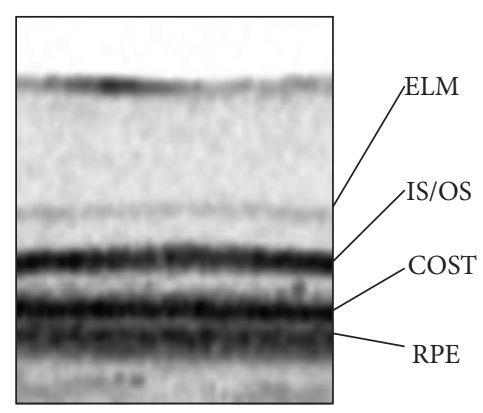

(b)

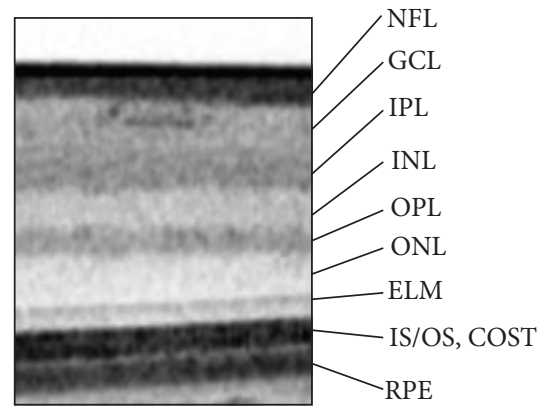

(c)

FIgure 1: (a) Retinal sections of the physiologic macula dissecting along the green line on fundus photography using SD-OCT. (b) The magnified image at the fovea shows three lines over the retinal pigment epithelium (RPE), that is, the external limiting membrane (ELM), the junction between inner and outer segments (IS/OS), and the cone outer segment tips (COST). (c) A magnified parafoveal image shows the individual retinal layers. NFL: nerve fiber layer; GCL: ganglion cell layer; IPL: inner plexiform layer; INL: inner nuclear layer; OPL: outer plexiform layer; ONL: outer nuclear layer.

quantification of the total retinal thickness (Figure 2). The diabetic retinopathy clinical research network (DRCRnet) especially defined an increase in the mean central thickness of $1 \mathrm{~mm}$ as center-involved DME [23,24], which is the new standard for applying treatments and a surrogate marker for evaluating treatment efficacy [25]. It is widely accepted that the central thickness is correlated modestly with visual acuity (VA) in DME [26]. In addition, the DRCRnet reported that paradoxical VA changes are observed after intervention, that is, VA reduction despite resolution of ME or VA improvement with increased retinal thickening [26]. These data suggest the importance of identifying unknown mechanisms and the magnitude of edematous changes. Despite these issues, an increasing number of studies have reported the relevance of measuring the retinal thickness after treatment for DME as a surrogate marker [27-29].

SD-OCT with higher resolution and reduced speckle noises has enabled segmentation of the individual retinal layers, and several kinds of OCT instruments provide automated segmentation. This feature has encouraged quantification of the thickness of the retinal layers and qualitative evaluation of lesions in the individual layers. Interestingly, the thickness in the inner retinal layers is correlated positively with visual impairment, whereas the outer retinal thickness is associated negatively with poor visual prognosis after vitrectomy for DME [30]. This suggests that thinning of the outer retinal layers is related to photoreceptor degeneration (or atrophy) concomitantly contributes to visual disturbance at least partly, and supports the paradoxical VA changes reported by the DRCRnet [26]. Further analyses of retinal thicknesses with segmentation would improve the understanding of the association between clinical findings and pathogenesis in DME.

\section{Pathomorphology in Individual Retinal Layers}

Numerous pathological mechanisms have been reported regarding diabetic retinopathy (DR), compared to the simple criteria of diabetes per se [1-4]. OCT subjectively shows several types of foveal morphologies in DME, that is, cystoid macular edema (CME), serous retinal detachment (SRD), and sponge-like retinal swelling [31], which might be among other factors that modulate visual function, dependently or independently of retinal thickening. Individual lesions have been delineated in the individual retinal layers. OCT showed cystoid spaces mainly in the INL and OPL, which has been supported by histologic reports [20,32,33]. Extracellular fluids pool between the outer segments and retinal pigment epithelium (RPE) in eyes with SRD. Sponge-like retinal swelling at the fovea occurs in the OPL.

Regarding the types of CME, OCT has documented several findings (Figure 3). The cystoid spaces in the INL 


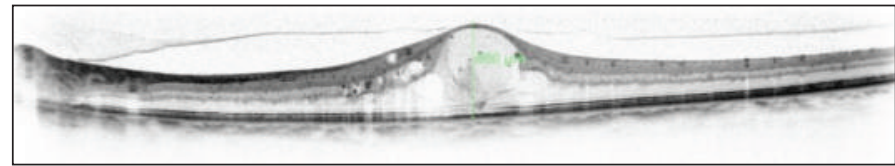

(a)

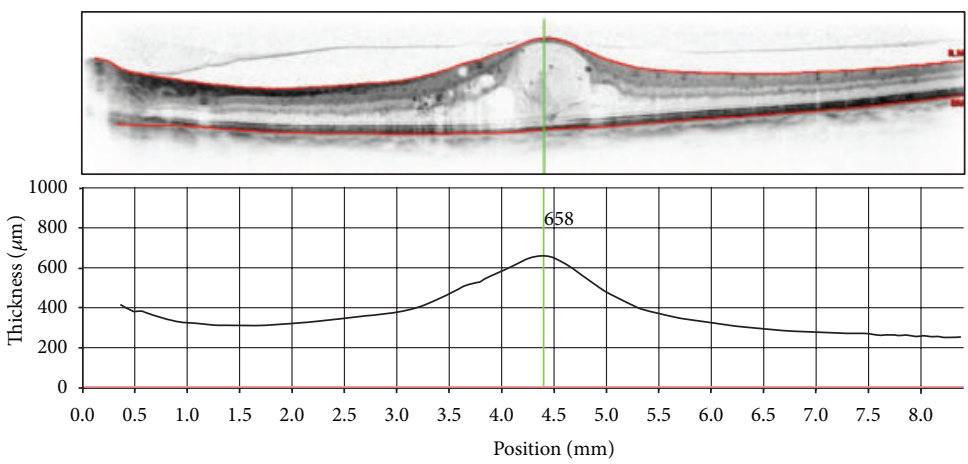

(b)

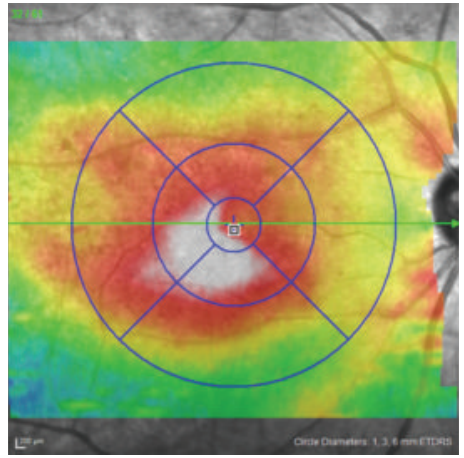

(c)

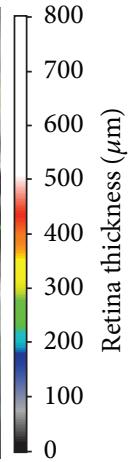

0

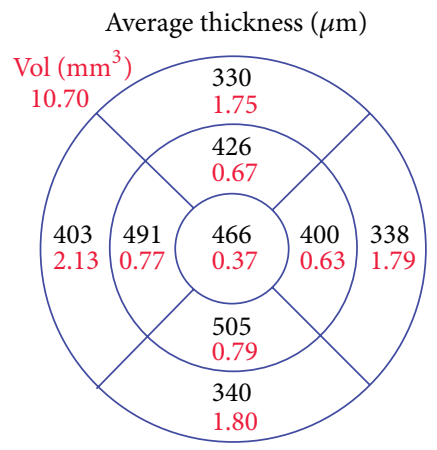

(d)

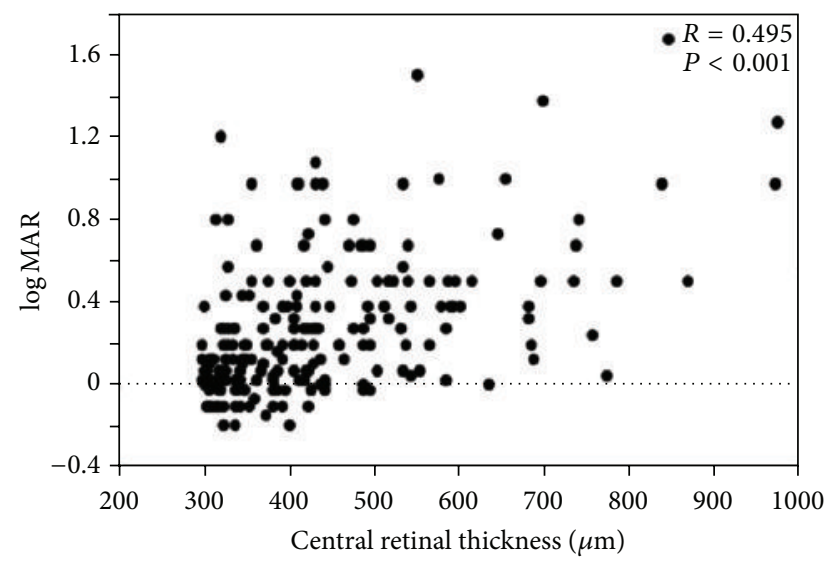

(e)

FIGURE 2: Quantification of the total retinal thickness using OCT. The retinal thickness can be measured semiautomatically (b) or manually using calipers (a). (c) A two-dimensional map of the retinal thickness can be constructed from the automatically measured retinal thickness. (d) The average thickness in each subfield of Early Treatment Diabetic Retinopathy Study grid is shown. (e) The average thickness in the central subfield is correlated modestly with the logarithm of the minimum angle of resolution VA in center-involved DME. 


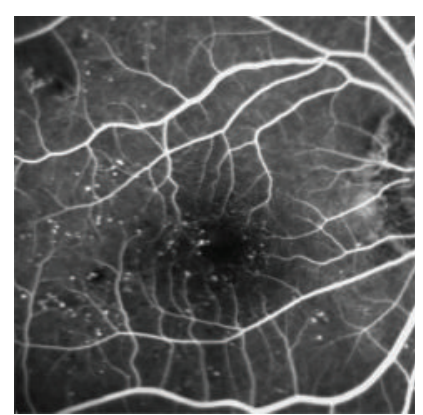

(a)

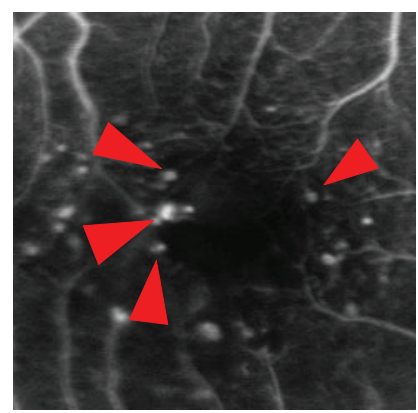

(b)

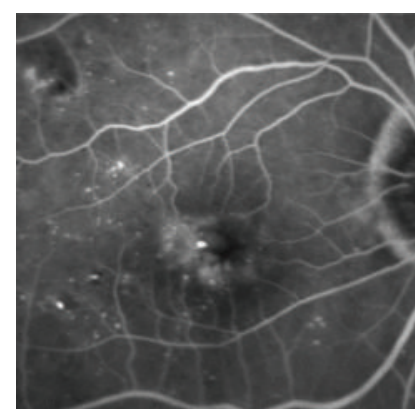

(c)
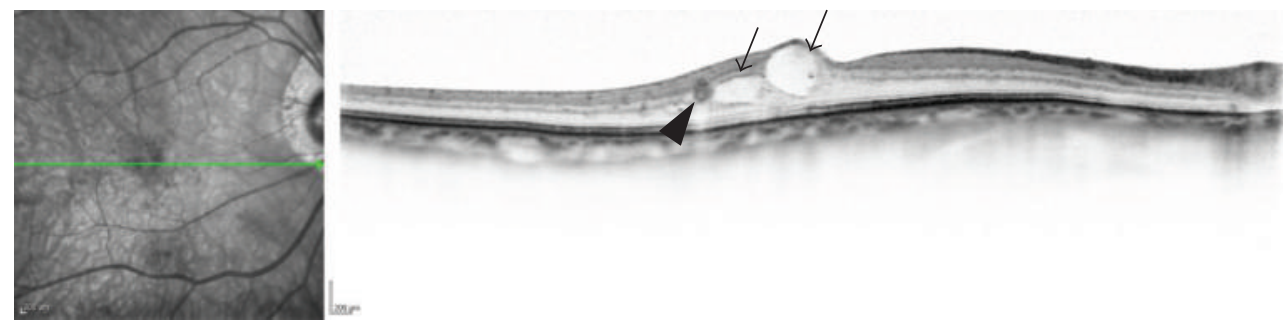

(d)

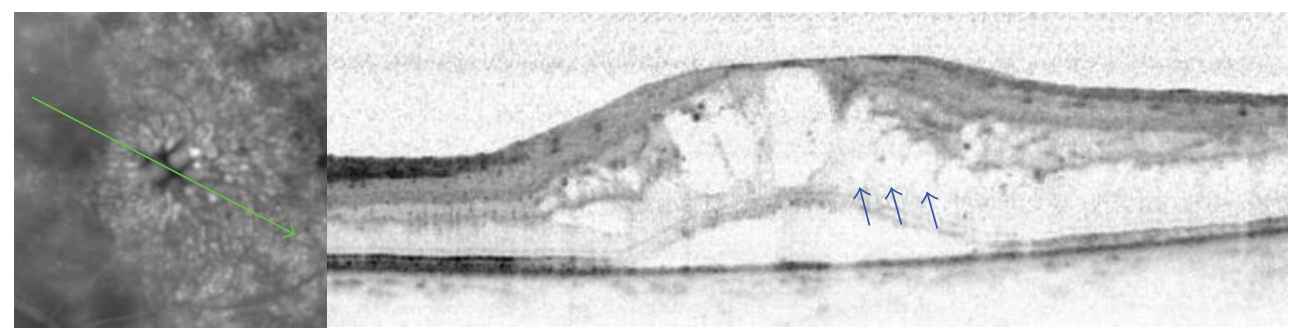

(e)
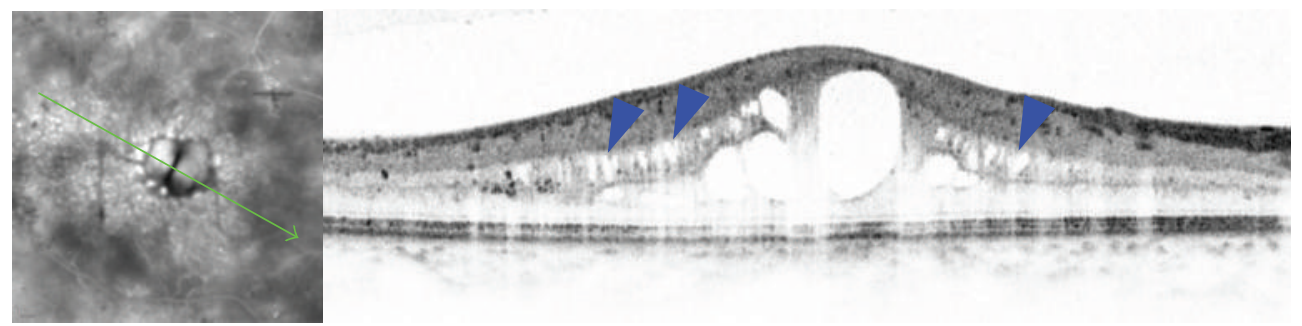

(f)

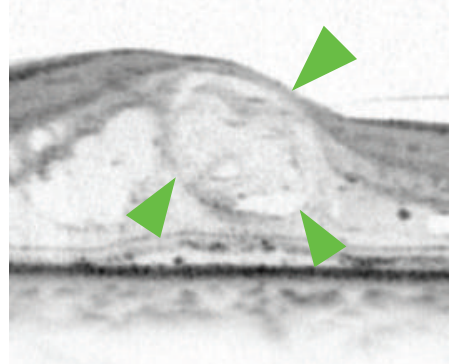

(g)

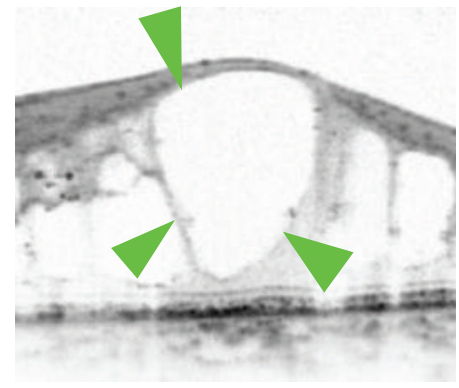

(h)

Figure 3: Characteristics of cystoid spaces in DME. ((a), (b)) Early-phase FA shows an enlarged foveal avascular zone and surrounding microaneurysms (arrowheads). (c) A late-phase image shows fluorescein pooling at the fovea. (d) Cystoid spaces (arrows) are accompanied by microaneurysms (arrowhead). (e) A late-phase FA image shows petalloid- (e) or honeycomb-pattern (f) fluorescein pooling, which is considered to correspond to cystoid spaces in OPL (arrow) or INL (arrowhead), respectively, on OCT image. Foveal cystoid spaces (arrowheads) have higher OCT reflectivity and its heterogeneity (g) or lower and homogeneous reflectivity (h). 
have a honeycomb pattern of fluorescein pooling, whereas petalloid-shaped pooling corresponds to cystoid spaces in the OPL $[32,33]$. Further study of an FA/OCT correlation found that the foveal cystoid spaces on OCT images were associated with enlarged foveal avascular zones and microaneurysms around the perifoveal capillary network [8]. This suggested that ischemia and leakage from microaneurysms contribute to the development or maintenance of cystoid spaces in DME. A recent publication reported that OCT reflectivity in the cystoid spaces was correlated negatively with the intensity of the fluorescein pooling [34] and implicated several types of vascular hyperpermeability regarding the pathogenesis in the cystoid spaces.

SD-OCT has shown the fine structures of the microaneurysms in patients with DR [35-37]. Among the capsular structure patterns, the "incomplete" and "absent" types, in contrast to the "complete" type, often were accompanied by fluorescein leakage and cystoid spaces [36, 37]. Recently, using SD-OCT, clinicians have confirmed that the number of microaneurysms decrease after focal photocoagulation, which suggested that in the future FA would be replaced with noninvasive OCT to evaluate treatment efficacy $[38,39]$.

Vitreomacular traction sometimes modulates macular thickening in DME [40-43]. Eliminating the vitreoretinal traction during vitrectomy is suggested to be an effective strategy for eyes with DME, and DRCRnet has reported the greater VA improvement in eyes with preoperative ERM and the greater reduction of central subfield thickness in eyes with vitreoretinal abnormalities [44-46]. Ocriplasmin, a recombinant protease with activity against components of the vitreoretinal interface, has recently been reported to be effective for the diseases with vitreomacular interface abnormalities [47]. It remains to be elucidated how ocriplasmin modulates retinal thickening without the removal of the vitreous gel which contains growth factors and cytokines.

Sponge-like retinal swelling especially often is accompanied by pathological findings in the vitreomacular interface on OCT images (Figure 4), and vitreomacular interface abnormalities contribute to thickening of the retinal parenchyma in the OPL at the fovea, as in the case of idiopathic epiretinal membrane (ERM). It also was reported that vitreomacular interface abnormalities also might induce SRD in DME [40], and OCT sometimes shows cystoid spaces with vitreoretinal abnormalities as with idiopathic macular holes $[48,49]$. Fibrovascular proliferation in PDR progresses along the posterior hyaloid membrane and induces contraction. Tangential tractional forces increase the retinal thickness and concomitantly contribute to macular edema, and horizontal forces result in macular heterotopia (traction maculopathy) in PDR. Several patterns of vitreomacular interface abnormalities seen on SD-OCT images were reported recently, that is, vitreomacular traction with no or partial posterior vitreous detachment, posterior vitreous separation, ERM, and their combinations. These findings would help surgeons to completely remove the pathological changes of the vitreoretinal interface [43].

It is not well known how SRD develops in DME compared to the pathogenesis in eyes with CME or sponge-like retinal swelling. Marmor reported numerous clinical and basic data regarding the development of retinal detachment that depended on the osmotic or oncotic pressure of intraocular fluids [50]. In eyes with DME, vascular hyperpermeability might increase such pressures, resulting in SRD. Highresolution OCT has enabled observation of the cystoid spaces in the OPL that sometimes rupture toward the SRD, suggesting that extravasated blood components pour directly into the SRD [51]. Regarding visual function, no association was found between VA and foveal thickness in eyes with foveal SRD [52], whereas these eyes often have a poor prognosis after treatment $[51,53]$. OCT often delineates hyperreflective foci in subretinal fluids (Figure 5). In most such cases, subfoveal hard exudates develop after resolution of the macular edema (ME) that correspond to the confluent hyperreflective foci with concomitant impaired visual function [51], as reported in the Early Treatment Diabetic Retinopathy Study [54, 55].

\section{Photoreceptor Layers}

The superior delineation of the fine structures on SDOCT images encouraged us to evaluate photoreceptor markers, external limiting membrane (ELM), and the junction between the inner and outer segments (IS/OS) (Figure 6). Sandberg and associates reported that the IS/OS line, to which they referred as the third high-reflectance band in their original manuscript, is associated with visual function in retinitis pigmentosa [56], suggesting that the IS/OS line represents the photoreceptor structure and function in vivo [57]. A few years later, disruption of the IS/OS line at the fovea was reported to be associated with a poor visual prognosis in resolved ME associated with branch retinal vein occlusion [58]. Many later cross-sectional or longitudinal studies have shown the clinical relevance of the IS/OS line in DME [59-67]. Histologic publications have reported "cystoid degeneration" in the photoreceptors at the fovea [68], which supports the disruption of the IS/OS line on OCT images. The thickness of the photoreceptor outer segments was quantified and found to be associated with visual function in DME [62]. The transverse length of the disrupted or absent IS/OS line also has been related to visual impairment [63, 66]. In the future, quantification of photoreceptor damage would improve the understanding of visual impairment in DME.

Despite the relevance, it is unknown whether the IS/OS line seen on OCT images truly corresponds to the histologic junction of the inner and outer segments. Spaide and Curcio speculated that this highly reflective band was located at the ellipsoid in the inner segments, considering the correlation between the microstructure on the SD-OCT images and the histologic findings [69]. The OCT reflectivity changed around the line after light exposure, which suggested that the line may represent photoreceptor function per se [70, 71].

The ELM line is another marker of photoreceptor integrity, and its disruption also is associated with visual impairment in DME [52, 66, 67]. Shin and associates reported that ELM disruption predicts poor visual outcomes after treatment with triamcinolone [67]. Since the ELM is an intercellular junction between the Müller cells and photoreceptor 


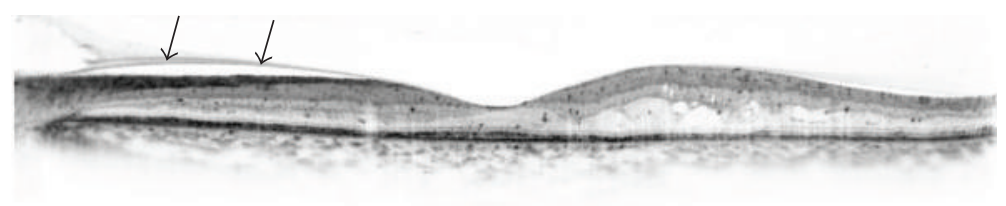

Les

(a)



Les

(b)

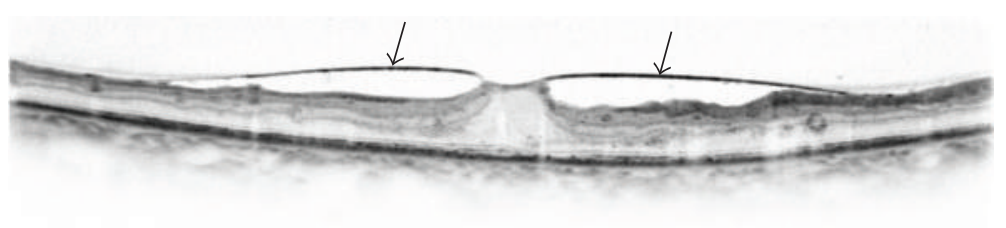

Les

(c)

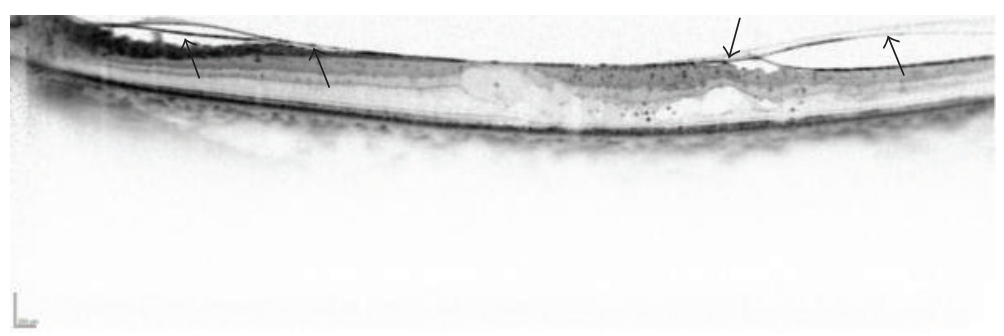

(d)

FIGURE 4: Several patterns of vitreomacular interface abnormalities in DME. (a) A posterior hyaloid membrane is sometimes depicted on OCT images (arrows). (b) ERM often is accompanied by sponge-like retinal swelling (arrows). (c) A perifoveal vitreous separation sometimes induces vertical traction, as in idiopathic macular holes (arrows). (d) Some eyes have separation of a posterior vitreous membrane (arrows).

cells and has barrier properties against macromolecules [72], the disrupted ELM might allow blood components to migrate into the outer retinal layers and exacerbate the photoreceptor damage. Although it remains poorly understood how the ELM becomes disrupted, a few possible mechanisms are implicated. Extended cystoid spaces from the INL to the OPL are accompanied by ELM disruption in DME, suggesting disturbance of the Müller cells [66]. A tear in the outer retinal layers also can result in loss of the barrier function in eyes with SRD [51].

SD-OCT shows dot-like lesions, referred to as hyperreflective foci, throughout the retina in DR [35]. Those in the outer layers especially are correlated cross-sectionally with poor visual function in patients with DME without SRD [73]. Hyperreflective foci in subretinal fluids accumulate at the fovea and lead to poor visual prognosis in eyes with SRD [51]. Bolz and associates speculated that the hyperreflective foci in DR corresponded to lipid-laden macrophages and the precursors of hard exudates [35]. The hyperreflective foci also are considered to be degenerated photoreceptors and RPE hyperplasia or metaplasia in other diseases [74, 75]. Although it remains undetermined what the hyperreflective foci are in the outer retinal layers in DME, the disruption of the ELM or IS/OS line is correlated with the hyperreflective foci, suggesting that these lesions reciprocally promote the pathogenesis of photoreceptor degeneration. 


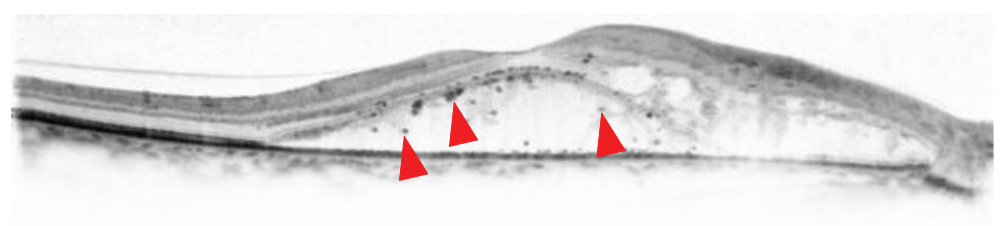

Las

(a)

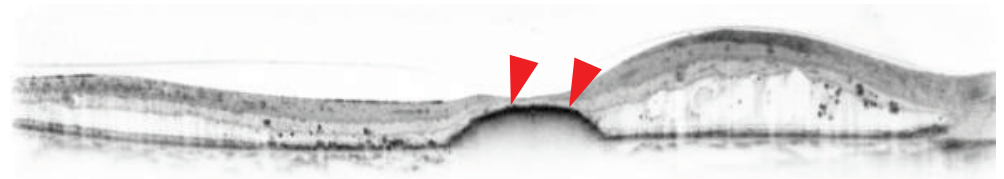

(b)

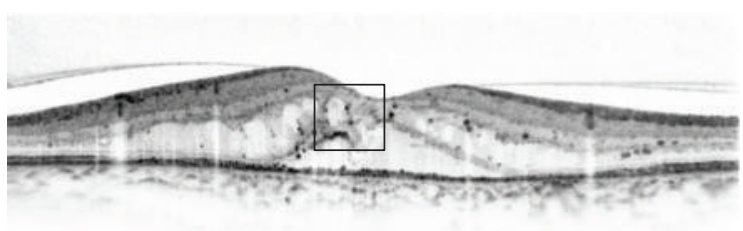

(c)

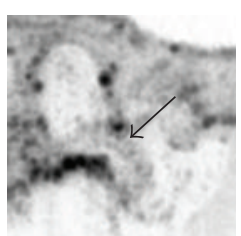

(d)

FIGURE 5: A representative case with hyperreflective foci (arrowheads) in subretinal fluids. The preoperative decimal VA is 0.6. (b) DME is improving after focal/grid photocoagulation, although hyperreflective foci have accumulated at the fovea (arrowheads). The postoperative VA is 0.09. (c) OCT sometimes shows that cystoid spaces in the OPL rupture to subretinal fluids (arrow), which might modulate the pathogenesis in SRD.

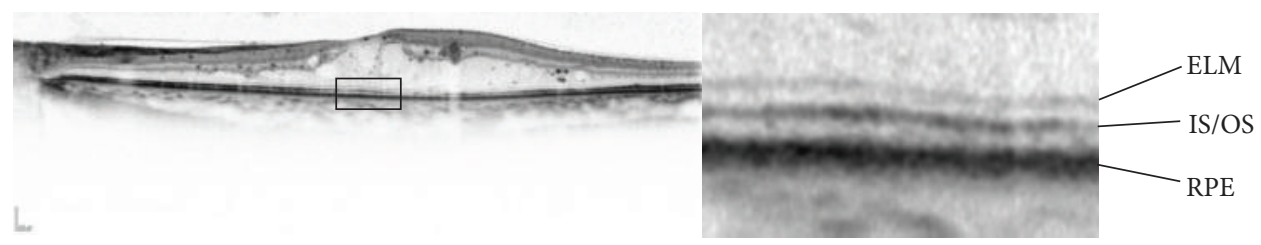

(a)

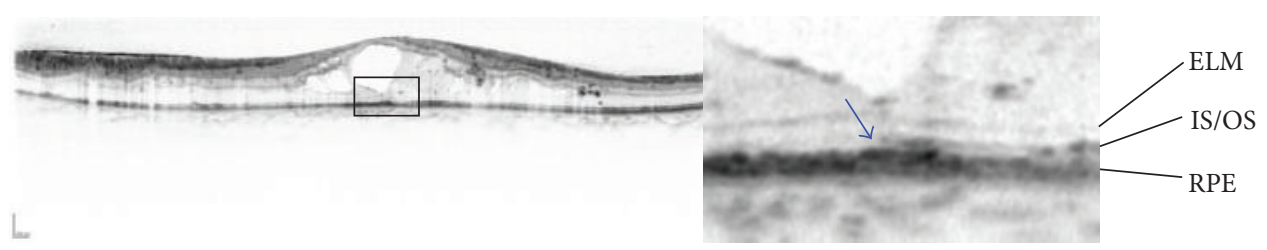

(b)

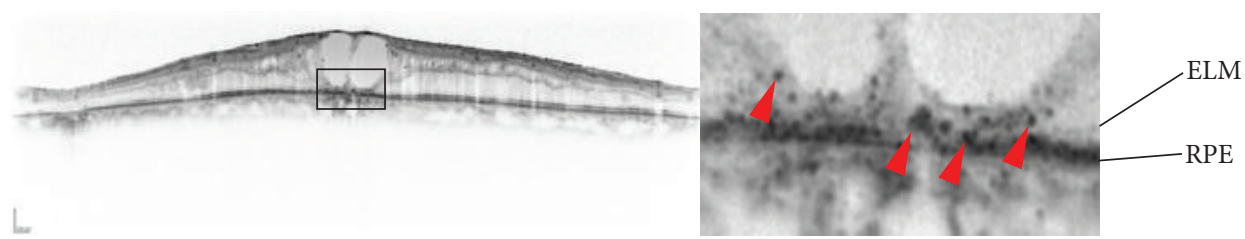

(c)

Figure 6: Pathological changes in the photoreceptor layers in DME. (a) Both the ELM and IS/OS lines are continuous. (b) The ELM line seems almost continuous, whereas the IS/OS line is discontinuous on the left (arrow). (c) The ELM line is disrupted, and the IS/OS line is absent at the fovea, accompanied by hyperreflective foci in the outer retinal layers (arrowheads). 


\section{Ganglion Cells and Nerve Fiber Layers}

The nerve fiber layer (NFL) is comprised of axons derived from ganglion cells. The defects in the NFL were clinically reported [76], and basic research showed ganglion cell apoptosis in DR [77]. Since swelling of the NFL occurs in lesions associated with DR, such as cotton-wool spots (soft exudates), it is difficult to evaluate the decrease in the axons from the ganglion cells using OCT. In contrast, thinning of the ganglion cell layer was reported in eyes with ischemic maculopathy with and without DME [78]. Further, glaucoma research has focused on the clinical relevance of the ganglion cell complex (from the inner limiting membrane to the outer boundary of the inner plexiform layer) [79], which should be applied to evaluate neovascular glaucoma in advanced PDR.

It was reported that white spots on fundus photography might correspond to hyperreflective lesions at the level of NFL [80]. Midena and associates have recently demonstrated that hyperreflective spots, which might correspond to activated microglia or Müller cells, were detected in inner retinal layers, as DR progresses. They were suggested to be a novel biomarker of glial activation in DR, and further investigation remains to be planned [81].

\section{The Choroid}

Disruption of the choroidal circulation in patients with diabetes had been reported as diabetic choroidopathy [82, 83]. Enhanced-depth imaging using SD-OCT or the latest version of OCT, swept-source OCT, recently has enabled measurement of the choroidal thickness. A few publications have reported alteration of the choroidal thickness [84-86] and further investigations will clarify how the pathological choroidal changes contribute to DME.

\section{Conclusions}

OCT has allowed identification of the morphologic factors in the pathogenesis in DME. The major OCT parameter, the central retinal thickness, is correlated modestly with the VA, and pathomorphologies and photoreceptor damage also cause visual impairment. Further studies of a structuralfunctional correlation will promote a better understanding of the complex pathogenesis in DME [87].

\section{Abbreviations}

CME: Cystoid macular edema

DME: Diabetic macular edema

DR: Diabetic retinopathy

ELM: External limiting membrane

ERM: Epiretinal membrane

FA: Fluorescein angiography

INL: Inner nuclear layer

IS/OS: The junction between inner and outer segments
ME: $\quad$ Macular edema

OPL: $\quad$ Outer plexiform layer

PDR: $\quad$ Proliferative diabetic retinopathy

SD-OCT: Spectral-domain optical coherence tomography

SRD: $\quad$ Serous retinal detachment.

\section{Conflict of Interests}

The authors declare that there is no conflict of interests.

\section{References}

[1] R. Klein, B. E. K. Klein, S. E. Moss, and K. J. Cruickshanks, "The Wisconsin epidemiologic study of diabetic retinopathy XV: the long- term incidence of macular edema," Ophthalmology, vol. 102, no. 1, pp. 7-16, 1995.

[2] T. W. Gardner, D. A. Antonetti, A. J. Barber, K. F. LaNoue, and S. W. Levison, "Diabetic retinopathy: more than meets the eye," Survey of Ophthalmology, vol. 47, supplement 2, pp. S253-S262, 2002.

[3] Q. Mohamed, M. C. Gillies, and T. Y. Wong, "Management of diabetic retinopathy: a systematic review," Journal of the American Medical Association, vol. 298, no. 8, pp. 902-916, 2007.

[4] D. A. Antonetti, R. Klein, and T. W. Gardner, "Diabetic retinopathy," The New England Journal of Medicine, vol. 366, no. 13, pp. 1227-1239, 2012.

[5] R. P. Phillips, P. G. Ross, M. Tyska, P. F. Sharp, and J. V. Forrester, "Detection and quantification of hyperfluorescent leakage by computer analysis of fundus fluorescein angiograms," Graefe's Archive for Clinical and Experimental Ophthalmology, vol. 229, no. 4, pp. 329-335, 1991.

[6] B. Sander, M. Larsen, B. Moldow, and H. Lund-Andersen, "Diabetic macular edema: passive and active transport of fluorescein through the blood-retina barrier," Investigative Ophthalmology \& Visual Science, vol. 42, no. 2, pp. 433-438, 2001.

[7] S. T. Knudsen, T. Bek, P. L. Poulsen, M. N. Hove, M. Rehling, and C. E. Mogensen, "Macular edema reflects generalized vascular hyperpermeability in type 2 diabetic patients with retinopathy," Diabetes Care, vol. 25, no. 12, pp. 2328-2334, 2002.

[8] T. Murakami, K. Nishijima, A. Sakamoto, M. Ota, T. Horii, and N. Yoshimura, "Foveal cystoid spaces are associated with enlarged foveal avascular zone and microaneurysms in diabetic macular edema," Ophthalmology, vol. 118, no. 2, pp. 359-367, 2011.

[9] A. M. Abu el Asrar, D. Maimone, P. H. Morse, S. Gregory, and A. T. Reder, "Cytokines in the vitreous of patients with proliferative diabetic retinopathy," American Journal of Ophthalmology, vol. 114, no. 6, pp. 731-736, 1992.

[10] A. P. Adamis, J. W. Miller, M.-T. Bernal et al., "Increased vascular endothelial growth factor levels in the vitreous of eyes with proliferative diabetic retinopathy," American Journal of Ophthalmology, vol. 118, no. 4, pp. 445-450, 1994.

[11] L. P. Aiello, R. L. Avery, P. G. Arrigg et al., "Vascular endothelial growth factor in ocular fluid of patients with diabetic retinopathy and other retinal disorders," The New England Journal of Medicine, vol. 331, no. 22, pp. 1480-1487, 1994.

[12] L. P. Aiello, S.-E. Bursell, A. Clermont et al., "Vascular endothelial growth factor-induced retinal permeability is mediated by protein kinase $\mathrm{C}$ in vivo and suppressed by an orally effective $\beta$-isoform-selective inhibitor," Diabetes, vol. 46, no. 9, pp. 14731480, 1997. 
[13] D. A. Antonetti, A. J. Barber, S. Khin, E. Lieth, J. M. Tarbell, and T. W. Gardner, "Vascular permeability in experimental diabetes is associated with reduced endothelial occludin content. Vascular endothelial growth factor decreases occludin in retinal endothelial cells. Penn State Retina Research Group," Diabetes, vol. 47, no. 12, pp. 1953-1959, 1998.

[14] E. T. Cunningham Jr., A. P. Adamis, M. Altaweel et al., "A phase II randomized double-masked trial of pegaptanib, an anti-vascular endothelial growth factor aptamer, for diabetic macular edema," Ophthalmology, vol. 112, no. 10, pp. 1747-1757, 2005.

[15] D. Watanabe, K. Suzuma, S. Matsui et al., "Erythropoietin as a retinal angiogenic factor in proliferative diabetic retinopathy," The New England Journal of Medicine, vol. 353, no. 8, pp. 782792, 2005.

[16] T. Murakami, E. A. Felinski, and D. A. Antonetti, "Occludin phosphorylation and ubiquitination regulate tight junction trafficking and vascular endothelial growth factor-induced permeability," Journal of Biological Chemistry, vol. 284, no. 31, pp. 21036-21046, 2009.

[17] M. Al-Shabrawey, R. Mussell, K. Kahook et al., "Increased expression and activity of 12-lipoxygenase in oxygen-induced ischemic retinopathy and proliferative diabetic retinopathy: implications in retinal neovascularization," Diabetes, vol. 60, no. 2, pp. 614-624, 2011.

[18] T. Murakami, T. Frey, C. Lin et al., "Protein kinase cbeta phosphorylates occludin regulating tight junction trafficking in vascular endothelial growth factor-induced permeability in vivo," Diabetes, vol. 61, no. 6, pp. 1573-1583, 2012.

[19] B. S. Fine and A. J. Brucker, "Macular edema and cystoid macular edema," American Journal of Ophthalmology, vol. 92, no. 4, pp. 466-481, 1981.

[20] M. O. M. Tso, "Pathology of cystoid macular edema," Ophthalmology, vol. 89, no. 8, pp. 902-915, 1982.

[21] M. Yanoff, B. S. Fine, A. J. Brucker, and R. C. Eagle Jr., "Pathology of human cystoid macular edema," Survey of Ophthalmology, vol. 28, supplement, pp. 505-511, 1984.

[22] D. Huang, E. A. Swanson, C. P. Lin et al., "Optical coherence tomography," Science, vol. 254, no. 5035, pp. 1178-1181, 1991.

[23] R. P. Danis, A. R. Glassman, L. P. Aiello et al., "Diurnal variation in retinal thickening measurement by optical coherence tomography in center-involved diabetic macular edema," Archives of Ophthalmology, vol. 124, no. 12, pp. 1701-1707, 2006.

[24] L. P. Aiello, R. W. Beck, N. M. Bressler et al., "Rationale for the diabetic retinopathy clinical research network treatment protocol for center-involved diabetic macular edema," Ophthalmology, vol. 118, no. e5, p. e14, 2011.

[25] K. G. Csaky, E. A. Richman, and F. L. Ferris III, "Report from the NEI/FDA ophthalmic clinical trial design and endpoints symposium," Investigative Ophthalmology \& Visual Science, vol. 49, no. 2, pp. 479-489, 2008.

[26] D. J. Browning, A. R. Glassman, L. P. Aiello et al., "Relationship between optical coherence tomography-measured central retinal thickness and visual acuity in diabetic macular edema," Ophthalmology, vol. 114, no. 3, pp. 525-536, 2007.

[27] T. Otani and S. Kishi, "Tomographic assessment of vitreous surgery for diabetic macular edema," American Journal of Ophthalmology, vol. 129, no. 4, pp. 487-494, 2000.

[28] M. J. Elman, L. P. Aiello, R. W. Beck et al., "Randomized trial evaluating ranibizumab plus prompt or deferred laser or triamcinolone plus prompt laser for diabetic macular edema," Ophthalmology, vol. 117, no. 6, pp. 1064-1077, 2010.
[29] M. Yoshikawa, T. Murakami, K. Nishijima et al., "Macular migration toward the optic disc after inner limiting membrane peeling for diabetic macular edema," Investigative Ophthalmology \& Visual Science, vol. 54, no. 1, pp. 629-635, 2013.

[30] T. Murakami, K. Nishijima, T. Akagi et al., "Segmentational analysis of retinal thickness after vitrectomy in diabetic macular edema," Investigative Ophthalmology \& Visual Science, vol. 53, no. 10, pp. 6668-6674, 2012.

[31] T. Otani, S. Kishi, and Y. Maruyama, "Patterns of diabetic macular edema with optical coherence tomography," American Journal of Ophthalmology, vol. 127, no. 6, pp. 688-693, 1999.

[32] T. Otani and S. Kishi, "Correlation between optical coherence tomography and fluorescein angiography findings in diabetic macular edema," Ophthalmology, vol. 114, no. 1, pp. 104-107, 2007.

[33] M. Bolz, M. Ritter, M. Schneider, C. Simader, C. Scholda, and U. Schmidt-Erfurth, "A systematic correlation of angiography and high-resolution optical coherence tomography in diabetic macular edema," Ophthalmology, vol. 116, no. 1, pp. 66-72, 2009.

[34] T. Horii, T. Murakami, K. Nishijima et al., "Relationship between fluorescein pooling and optical coherence tomographic reflectivity of cystoid spaces in diabetic macular edema," Ophthalmology, vol. 119, no. 5, pp. 1047-1055, 2012.

[35] M. Bolz, U. Schmidt-Erfurth, G. Deak, G. Mylonas, K. Kriechbaum, and C. Scholda, "Optical coherence tomographic hyperreflective foci: a morphologic sign of lipid extravasation in diabetic macular edema," Ophthalmology, vol. 116, no. 5, pp. 914-920, 2009.

[36] T. Horii, T. Murakami, K. Nishijima, A. Sakamoto, M. Ota, and N. Yoshimura, "Optical coherence tomographic characteristics of microaneurysms in diabetic retinopathy," American Journal of Ophthalmology, vol. 150, no. 6, pp. 840-848, 2010.

[37] H. Ito, T. Horii, K. Nishijima et al., "Association between fluorescein leakage and optical coherence tomographic characteristics of microaneurysms in diabetic retinopathy," Retina, vol. 33, no. 4, pp. 732-739, 2013.

[38] S. N. Lee, J. Chhablani, C. K. Chan et al., "Characterization of microaneurysm closure after focal laser photocoagulation in diabetic macular edema," American Journal of Ophthalmology, vol. 155, no. 5, pp. 905-912, 2013.

[39] Y. Yamada, K. Suzuma, A. Fujikawa et al., "Imaging of laserphotocoagulated diabetic microaneurysm with spectral domain optical coherence tomography," Retina, vol. 33, no. 4, pp. $726-$ 731, 2013.

[40] P. K. Kaiser, C. D. Riemann, J. E. Sears, and H. Lewis, "Macular traction detachment and diabetic macular edema associated with posterior hyaloidal traction," American Journal of Ophthalmology, vol. 131, no. 1, pp. 44-49, 2001.

[41] A. Gandorfer, M. Rohleder, S. Grosselfinger, C. Haritoglou, M. Ulbig, and A. Kampik, "Epiretinal pathology of diffuse diabetic macular edema associated with vitreomacular traction," American Journal of Ophthalmology, vol. 139, no. 4, pp. 638-652, 2005.

[42] D. Gaucher, R. Tadayoni, A. Erginay, B. Haouchine, A. Gaudric, and P. Massin, "Optical coherence tomography assessment of the vitreoretinal relationship in diabetic macular edema," American Journal of Ophthalmology, vol. 139, no. 5, pp. 807-813, 2005.

[43] A. Ophir and M. R. Martinez, "Epiretinal membranes and incomplete posterior vitreous detachment in diabetic macular edema, detected by spectral-domain optical coherence tomography," Investigative Ophthalmology \& Visual Science, vol. 52, no. 9, pp. 6414-6420, 2011. 
[44] H. Lewis, G. W. Abrams, M. S. Blumenkranz, and R. V. Campo, "Vitrectomy for diabetic macular traction and edema associated with posterior hyaloidal traction," Ophthalmology, vol. 99, no. 5, pp. 753-759, 1992.

[45] C. J. Flaxel, A. R. Edwards, L. P. Aiello et al., "Factors associated with visual acuity outcomes after vitrectomy for diabetic macular edema: diabetic retinopathy clinical research network," Retina, vol. 30, no. 9, pp. 1488-1495, 2010.

[46] J. A. Haller, H. Qin, R. S. Apte et al., "Vitrectomy outcomes in eyes with diabetic macular edema and vitreomacular traction," Ophthalmology, vol. 117, no. 6, pp. 1087-1093, 2010.

[47] P. Stalmans, M. S. Benz, A. Gandorfer et al., "Enzymatic vitreolysis with ocriplasmin for vitreomacular traction and macular holes," The New England Journal of Medicine, vol. 367, no. 7, pp. 606-615, 2012.

[48] M. Hangai, Y. Ojima, N. Gotoh et al., "Three-dimensional imaging of macular holes with high-speed optical coherence tomography," Ophthalmology, vol. 114, no. 4, pp. 763-773, 2007.

[49] A. Takahashi, T. Nagaoka, S. Ishiko, D. Kameyama, and A. Yoshida, "Foveal anatomic changes in a progressing stage $1 \mathrm{mac}-$ ular hole documented by spectral-domain optical coherence tomography," Ophthalmology, vol. 117, no. 4, pp. 806-810, 2010.

[50] M. F. Marmor, "Mechanisms of fluid accumulation in retinal edema," Documenta Ophthalmologica, vol. 97, no. 3-4, pp. 239249, 1999.

[51] M. Ota, K. Nishijima, A. Sakamoto et al., "Optical coherence tomographic evaluation of foveal hard exudates in patients with diabetic maculopathy accompanying macular detachment," Ophthalmology, vol. 117, no. 10, pp. 1996-2002, 2010.

[52] T. Murakami, K. Nishijima, A. Sakamoto, M. Ota, T. Horii, and N. Yoshimura, "Association of pathomorphology, photoreceptor status, and retinal thickness with visual acuity in diabetic retinopathy," American Journal of Ophthalmology, vol. 151, no. 2, pp. 310-317, 2011.

[53] M. Shimura, K. Yasuda, T. Nakazawa et al., "Visual outcome after intravitreal triamcinolone acetonide depends on optical coherence tomographic patterns in patients with diffuse diabetic macular edema," Retina, vol. 31, no. 4, pp. 748-754, 2011.

[54] E. Y. Chew, M. L. Klein, F. L. Ferris III et al., "Association of elevated serum lipid levels with retinal hard exudate in diabetic retinopathy. Early Treatment Diabetic Retinopathy Study (ETDRS) Report 22," Archives of Ophthalmology, vol. 114, no. 9, pp. 1079-1084, 1996.

[55] D. S. Fong, P. P. Segal, F. Myers, F. L. Ferris, L. D. Hubbard, and M. D. Davis, "Subretinal fibrosis in diabetic macular edema. ETDRS report 23. Early Treatment Diabetic Retinopathy Study Research Group," Archives of Ophthalmology, vol. 115, no. 7, pp. 873-877, 1997.

[56] M. A. Sandberg, R. J. Brockhurst, A. R. Gaudio, and E. L. Berson, "The association between visual acuity and central retinal thickness in retinitis pigmentosa," Investigative Ophthalmology \& Visual Science, vol. 46, no. 9, pp. 3349-3354, 2005.

[57] T. Murakami, M. Akimoto, S. Ooto et al., "Association between abnormal autofluorescence and photoreceptor disorganization in retinitis pigmentosa," American Journal of Ophthalmology, vol. 145, no. 4, pp. 687-694, 2008.

[58] T. Murakami, A. Tsujikawa, M. Ohta et al., "Photoreceptor status after resolved macular edema in branch retinal vein occlusion treated with tissue plasminogen activator," American Journal of Ophthalmology, vol. 143, no. 1, pp. 171-173, 2007.

[59] G. Querques, A. V. Bux, D. Martinelli, C. Iaculli, and N. D. Noci, "Intravitreal pegaptanib sodium (Macugen) for diabetic macular oedema," Acta Ophthalmologica, vol. 87, no. 6, pp. 623630, 2009.

[60] A. Sakamoto, K. Nishijima, M. Kita, H. Oh, A. Tsujikawa, and N. Yoshimura, "Association between foveal photoreceptor status and visual acuity after resolution of diabetic macular edema by pars plana vitrectomy," Graefe's Archive for Clinical and Experimental Ophthalmology, vol. 247, no. 10, pp. 1325-1330, 2009.

[61] T. Alasil, P. A. Keane, J. F. Updike et al., "Relationship between optical coherence tomography retinal parameters and visual acuity in diabetic macular edema," Ophthalmology, vol. 117, no. 12, pp. 2379-2386, 2010.

[62] F. Forooghian, P. F. Stetson, S. A. Meyer et al., "Relationship between photoreceptor outer segment length and visual acuity in diabetic macular edema," Retina, vol. 30, no. 1, pp. 63-70, 2010.

[63] A. S. Maheshwary, S. F. Oster, R. M. S. Yuson, L. Cheng, F. Mojana, and W. R. Freeman, "The association between percent disruption of the photoreceptor inner segment-outer segment junction and visual acuity in diabetic macular edema," American Journal of Ophthalmology, vol. 150, no. 1, pp. 63-67, 2010.

[64] T. Otani, Y. Yamaguchi, and S. Kishi, "Correlation between visual acuity and foveal microstructural changes in diabetic macular edema," Retina, vol. 30, no. 5, pp. 774-780, 2010.

[65] A. Yanyali, K. T. Bozkurt, A. Macin, F. Horozoglu, and A. F. Nohutcu, "Quantitative assessment of photoreceptor layer in eyes with resolved edema after pars plana vitrectomy with internal limiting membrane removal for diabetic macular edema," Ophthalmologica, vol. 226, no. 2, pp. 57-63, 2011.

[66] T. Murakami, K. Nishijima, T. Akagi et al., "Optical coherence tomographic reflectivity of photoreceptors beneath cystoid spaces in diabetic macular edema," Investigative Ophthalmology \& Visual Science, vol. 53, no. 3, pp. 1506-1511, 2012.

[67] H. J. Shin, S. H. Lee, H. Chung, and H. C. Kim, "Association between photoreceptor integrity and visualoutcome in diabetic macular edema," Graefe's Archive for Clinical and Experimental Ophthalmology, vol. 250, no. 1, pp. 61-70, 2012.

[68] I. H. L. Wallow, R. P. Danis, C. Bindley, and M. Neider, "Cystoid macular degeneration in experimental branch retinal vein occlusion," Ophthalmology, vol. 95, no. 10, pp. 1371-1379, 1988.

[69] R. F. Spaide and C. A. Curcio, "Anatomical correlates to the bands seen in the outer retina by optical coherence tomography: literature review and model," Retina, vol. 31, no. 8, pp. 1609-1619, 2011.

[70] K. Bizheva, R. Pflug, B. Hermann et al., "Optophysiology: depth-resolved probing of retinal physiology with functional ultrahigh-resolution optical tomography," Proceedings of the National Academy of Sciences of the United States of America, vol. 103, no. 13, pp. 5066-5071, 2006.

[71] V. J. Srinivasan, M. Wojtkowski, J. G. Fujimoto, and J. S. Duker, "In vivo measurement of retinal physiology with high-speed ultrahigh-resolution optical coherence tomography," Optics Letters, vol. 31, no. 15, pp. 2308-2310, 2006.

[72] A. H. Bunt-Milam, J. C. Saari, I. B. Klock, and G. G. Garwin, "Zonulae adherentes pore size in the external limiting membrane of the rabbit retina," Investigative Ophthalmology \& Visual Science, vol. 26, no. 10, pp. 1377-1380, 1985.

[73] A. Uji, T. Murakami, K. Nishijima et al., "Association between hyperreflective foci in the outer retina, status of photoreceptor 
layer, and visual acuity in diabetic macular edema," American Journal of Ophthalmology, vol. 153, no. 4, pp. 710-717, 2012.

[74] S. G. Schuman, A. F. Koreishi, S. Farsiu, S.-H. Jung, J. A. Izatt, and C. A. Toth, "Photoreceptor layer thinning over drusen in eyes with age-related macular degeneration imaged in vivo with spectral-domain optical coherence tomography," Ophthalmology, vol. 116, no. 3, pp. 488-496, 2009.

[75] S. Baumüller, P. C. Issa, H. P. N. Scholl, S. Schmitz-Valckenberg, and F. G. Holz, "Outer retinal hyperreflective spots on spectraldomain optical coherence tomography in macular telangiectasia type 2," Ophthalmology, vol. 117, no. 11, pp. 2162-2168, 2010.

[76] E. Chihara, T. Matsuoka, Y. Ogura, and M. Matsumura, "Retinal nerve fiber layer defect as an early manifestation of diabetic retinopathy," Ophthalmology, vol. 100, no. 8, pp. 1147-1151, 1993.

[77] A. J. Barber, E. Lieth, S. A. Khin, D. A. Antonetti, A. G. Buchanan, and T. W. Gardner, "Neural apoptosis in the retina during experimental and human diabetes: early onset and effect of insulin," Journal of Clinical Investigation, vol. 102, no. 4, pp. 783-791, 1998.

[78] S. H. Byeon, Y. K. Chu, H. Lee, S. Y. Lee, and O. W. Kwon, "Foveal ganglion cell layer damage in ischemic diabetic maculopathy: correlation of optical coherence tomographic and anatomic changes," Ophthalmology, vol. 116, no. 10, pp. 1949-1959, 2009.

[79] O. Tan, V. Chopra, A. T.-H. Lu et al., "Detection of macular ganglion cell loss in glaucoma by Fourier-domain optical coherence tomography," Ophthalmology, vol. 116, no. 12, pp. 2305-2314, 2009.

[80] H. W. van Dijk, P. H. Kok, M. E. van Velthoven et al., "White spots in the macula of patients with diabetes mellitus type 1 , without or with minimal diabetic retinopathy," Investigative Ophthalmology \& Visual Science, vol. 49, 2008.

[81] G. Midena, S. Bini, M. Berton, R. Parrozzani, S. Vujosevic, and E. Midena, "Retinal microglia activity mirrors the progression of diabetic retinopathy. An in vivo spectral domain OCT study," Investigative Ophthalmology \& Visual Science, vol. 54, 2013.

[82] J. B. Saracco, P. Gastaud, B. Ridings, and C. A. Ubaud, "Diabetic choroidopathy," Journal Francais d'Ophtalmologie, vol. 5, no. 4, pp. 231-236, 1982.

[83] A. A. Hidayat and B. S. Fine, "Diabetic choroidopathy: light and electron microscopic observations of seven cases," Ophthalmology, vol. 92, no. 4, pp. 512-522, 1985.

[84] M. Esmaeelpour, B. Považay, B. Hermann et al., "Mapping choroidal and retinal thickness variation in type 2 diabetes using three-dimensional 1060-nm optical coherence tomography," Investigative Ophthalmology \& Visual Science, vol. 52, no. 8, pp. 5311-5316, 2011.

[85] G. Querques, R. Lattanzio, L. Querques et al., "Enhanced depth imaging optical coherence tomography in type 2 diabetes," Investigative Ophthalmology \& Visual Science, vol. 53, no. 10, pp. 6017-6024, 2012.

[86] S. Vujosevic, F. Martini, F. Cavarzeran, E. Pilotto, and E. Midena, "Macular and peripapillary choroidal thickness in diabetic patients," Retina, vol. 32, no. 9, pp. 1781-1790, 2012.

[87] S. Vujosevic, E. Midena, E. Pilotto, P. P. Radin, L. Chiesa, and F. Cavarzeran, "Diabetic macular edema: correlation between microperimetry and optical coherence tomography findings," Investigative Ophthalmology \& Visual Science, vol. 47, no. 7, pp. 3044-3051, 2006. 


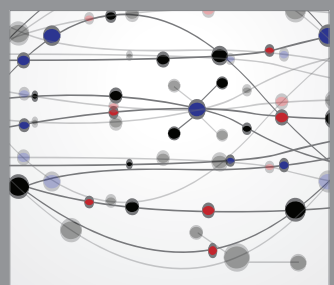

The Scientific World Journal
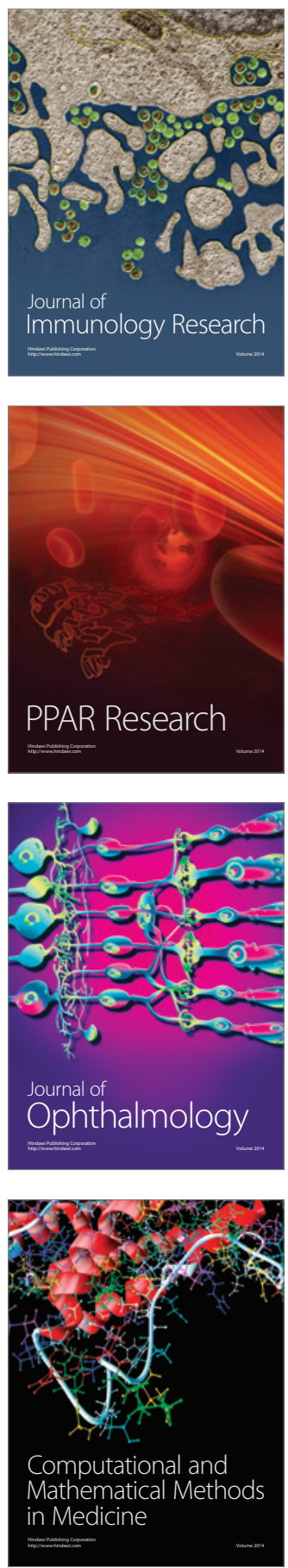

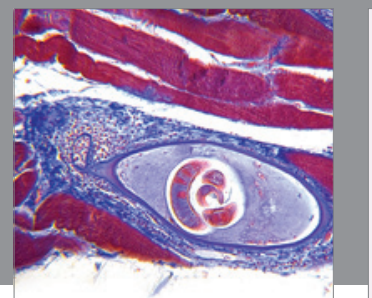

Gastroenterology

Research and Practice
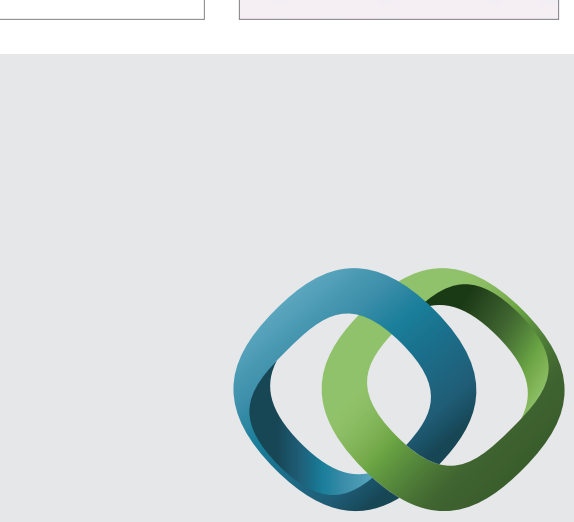

\section{Hindawi}

Submit your manuscripts at

http://www.hindawi.com
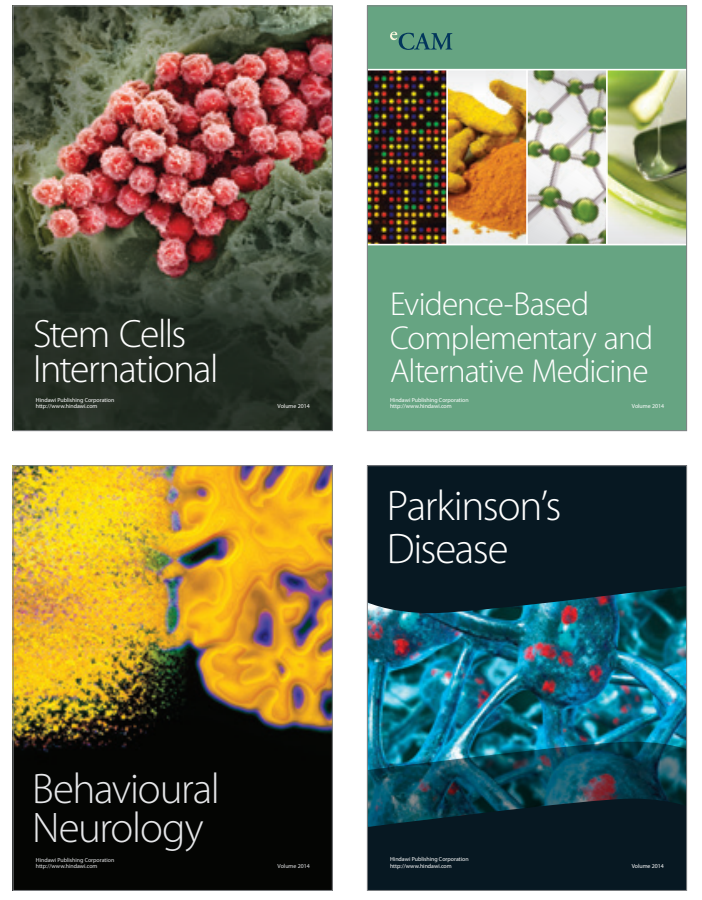
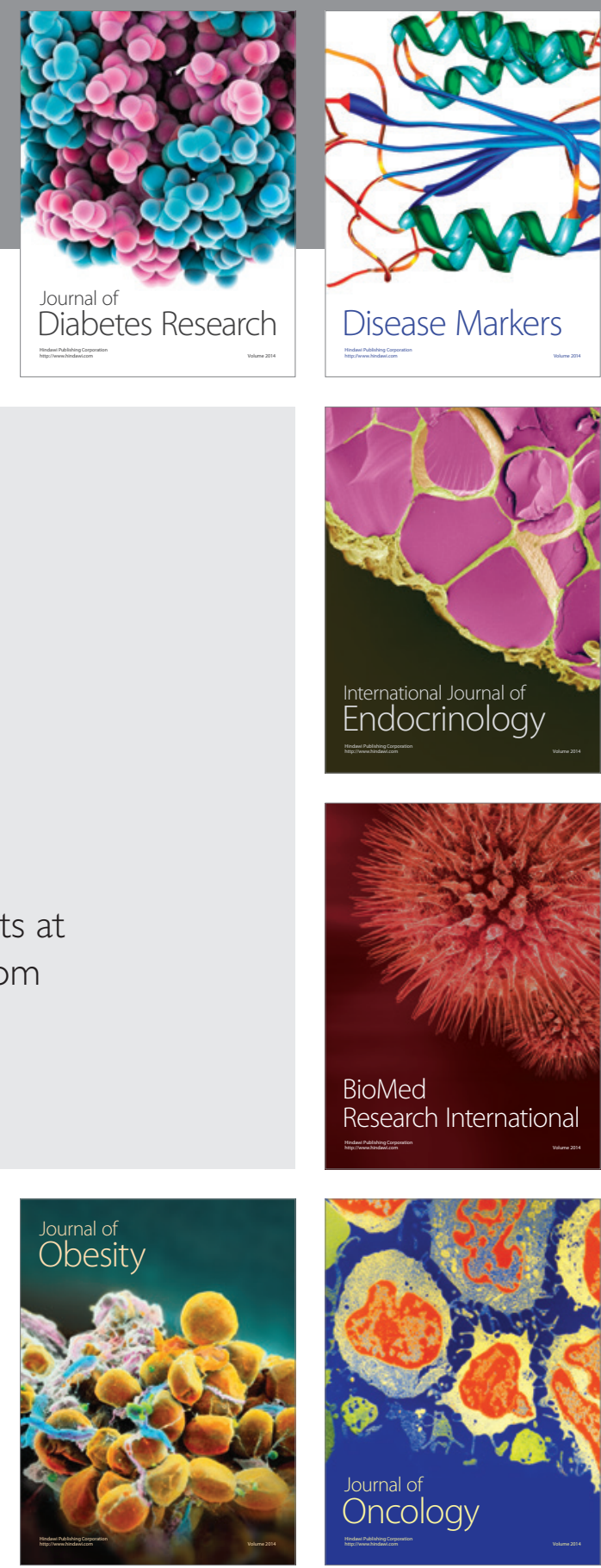

Disease Markers
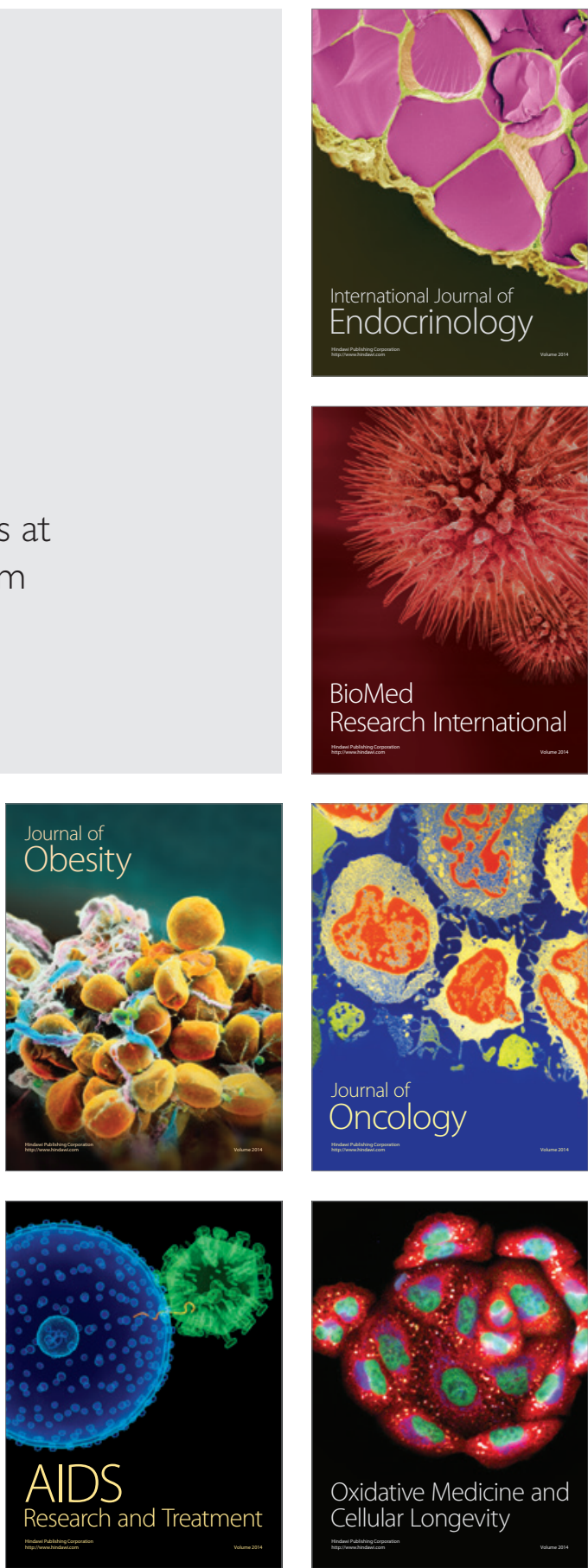